\title{
Instinctive drift in nondomesticated rodents
}

\author{
ROBERT W. POWELL and MICHAEL CURLEY \\ University of South Florida, Tampa, Florida 99620
}

\begin{abstract}
In the first experiment, Mongolian gerbils were studied under gradually increasing and decreasing fixed-ratio schedules of food reinforcement. Most of the animals showed a permanent displacement of the leverpress response by more genetically based behaviors, such as scratching and biting, as the ratio requirement increased. This type of phenomenon has been referred to as instinctive drift. The change in response topography resulted in high response rates and brief postreinforcement pauses, which remained relatively constant despite changes in the ratio. The few animals that maintained the leverpress response, which had initially been shaped, showed the usual direct relationship between ratio size and duration of the postreinforcement pause. The above effects were independent of the type of manipulandum, which included an omnidirectional lever, a conventional rectangular lever, and a metal chain suspended from the ceiling of the test chamber. In the second experiment, a group of nondomesticated cotton rats was studied under conditions similar to those employed in Experiment 1, except that higher ratios were included. All of these animals quickly developed instinctive drift, with the leverpress being displaced by scratching and biting. Response topographies of the rats were quite similar to those displayed earlier by the majority of gerbils. The duration of postreinforcement pauses remained relatively constant as the ratio increased, except for a marked increase in the mean pause at the highest ratio (90) studied. The overall results suggest that a dual system of control may operate during appetitive schedules, with responding being influenced by both elicitation and reinforcement mechanisms.
\end{abstract}

Breland and Breland (1961) coined the term "instinctive drift" to refer to the displacement of arbitrary conditioned responses by more genetically based behaviors, which are associated with food getting in the natural environment of the animal. In their attempts to train a variety of species, they had frequently observed this phenomenon. As an example, they cite the case of a raczoon which was easily trained to pick up a coin, carry it to a metal box, and deposit it in a slot for food reinforcement. However, when the animal was required to do the same thing with two coins, it persisted in rubbing them together and dipping the coins into the box, but frequently would not let go of them. This resulted in nonreinforcement, but, despite this fact, the rubbing and dipping occurred more frequently over time. These behaviors appeared similar to responses associated with feeding in the natural environment of the raccoon.

With the recent resurgence of interest in the biology of behavior among experimental psychologists, the concept of instinctive drift has received considerable attention, particularly in regard to its theoretical implications (Bolles, 1972; Staddon \& Simmelhag, 1971). However, other than the Breland's report, which was entirely anecdotal, there is little empirical evidence bearing on the validity of this concept.

It was not our initial purpose to study instinctive drift. Rather, we sought to extend the generality of the direct relationship which has been found to exist between the size of a fixed-ratio (FR) schedule of food reinforcement and the duration of the pauses which follow food delivery (Felton \& Lyon, 1966; Ferster \& Skinner, 1957; Powell, 1968, 1972). When we observed shanges in the topography of the responses in our first group of subjects that were correlated with a relatively constant postreinforcement pause (PRP) regardless of the FR size, we decided to replicate our study with additional subjects and different manipulanda. We suspected that the manipulandum used with our first group, an omnidirectional lever, might have interacted idiosyncratically with the behavior of the gerbil to foster apparent instinctive drift.

\section{EXPERIMENT I}

\section{Method}

Subjects. The subjects were eight female and six male Mongolian gerbils, between 1 and 2 years of age, maintained at approximately $80 \%$ of their normal weights during the experiment.

Apparatus. A conventional operant conditioning test chamber, $30 \times 20 \times 23 \mathrm{~cm}$, housed within a sound-attenuating chamber, was used. The test chamber contained a foodcup mounted on one wall, $.5 \mathrm{~cm}$ above the grid floor. Noyes pellets $(45 \mathrm{mg})$ served as the reinforcer. One of three manipulanda was present during successive phases of the experiment. The manipulanda used were a round plastic omnidirectional lever, a rectangular metal lever, and a metal chain which was suspended from the ceiling of the test chamber. Each of the levers was mounted on the same wall as the foodcup, and $4.0 \mathrm{~cm}$ from it. Illumination was provided by a $7-\mathrm{W}$ yellow houselight. Schedule programming, delivery of reinforcers, and recording of responses were accomplished by conventional electromechanical equipment. Direct observations of response topography were made during each experimental session.

Procedure. The experimental program was a systematic replication of an earlier experiment in our laboratory which had employed pigeons (Powell, 1968). When evidence of instinctive drift was found in the first group of six subjects, studied with an omnidirectional lever, we extended the study to include two additional groups of six and five subjects each, studied with a rec- 


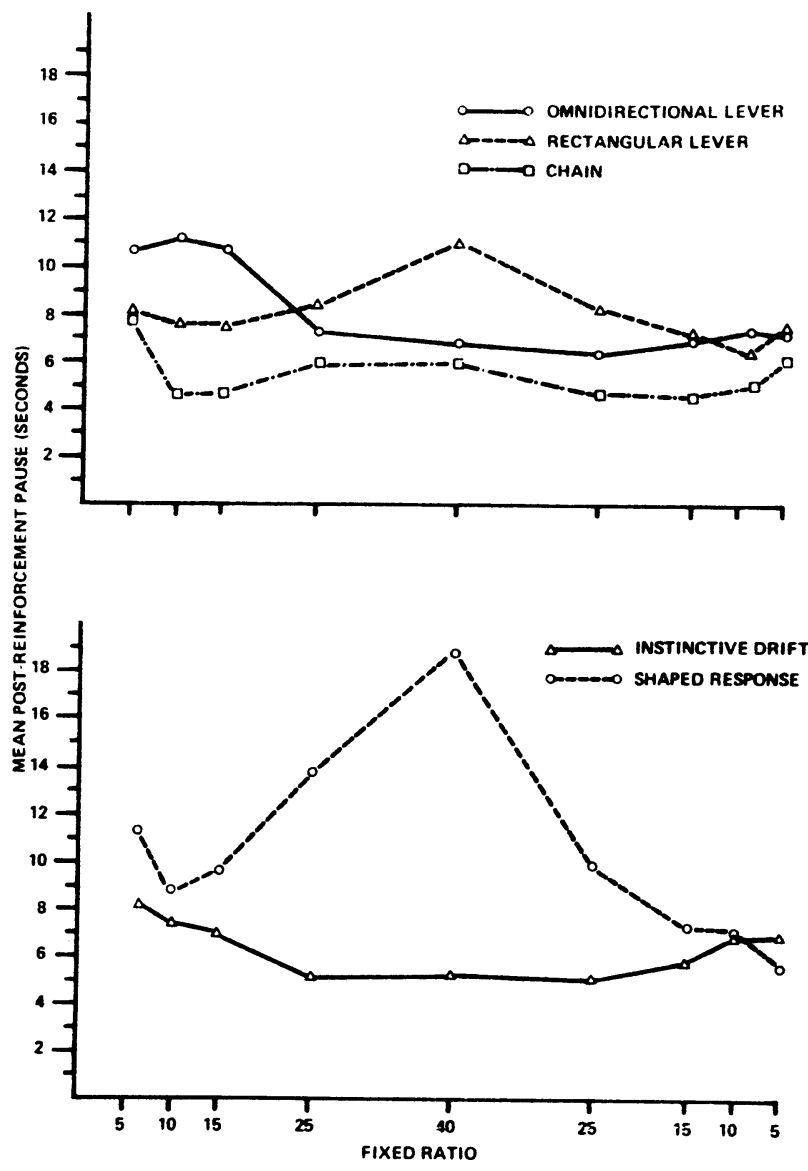

Figure 1. Upper portion: The mean postreinforcement pause for each manipulandum group as a function of the FR requirement. Each data point represents the mean performance over the final three sessions at each FR. Lower portion: The same data as presented above except that the gerbils are divided into two groups based upon whether the animal showed instinctive drift or maintained the leverpress response.

tangular lever and a metal chain, respectively. The latter group included three gerbils that had been studied first with the rectangular lever. With this exception, all of the gerbils were experimentally naive prior to FR training. The gerbils were shaped first by successive approximation to make the required operant response. All subjects were exposed to a schedule of increasing and decreasing FR requirements, the highest of which was FR-40. Each ratio requirement remained in effect for 5 sessions for all groups, except that FR -40 continued for 10 sessions for subjects making the chain-pull response. This was done to allow additional time for responding to stabilize. The duration of a session was based on the time it took each gerbil to obtain 34 reinforcers.

\section{Results}

Although all of the gerbils were initially shaped to press downward with two paws upon the levers, or to pull the chain downward with two paws, 10 of the 14 gerbils studied showed a substantial change in response topography during the experiment. For these animals, the operant response was replaced by behaviors which appear to be part of the animal's genetically based response repertoire. This typically occurred at FR-10 o FR-15. Once the change in response topograph: occurred (instinctive drift), the original operant respons did not reappear, even when the FR decreased. With th appearance of instinctive drift, PRPs showed littl change as FR size increased, and then decreased. Thus the PRP curves for the three groups are relatively flat, a: shown in the upper portion of Figure 1.

Five of the six gerbils studied with the omnidirec tional lever showed instinctive drift. These animals devel oped rapid scratching and biting upon the lever, behavio which appeared topographically similar to burrowing Four of the six gerbils studied with the rectangular leve also displayed instinctive drift, with their response being quite similar to the behavior described above. Th, five gerbils trained to respond by chain-pulling includer three animals that had displayed instinctive drift whe1 studied with the rectangular lever. The gerbils in thi group were shaped to grasp the chain with two paws anc pull it downward a minimum of $1.0 \mathrm{~cm}$. The respons topography of all animals, including the three gerbil studied earlier, appeared similar following shaping. Fou of the five gerbils in this group subsequently displayer instinctive drift. The predominant form of respons which developed was for the gerbil to grasp the chais with both paws, tilt its head to a 90-deg angle, and gnav the chain. The gerbils also rotated the chain as the gnawed upon it. The three gerbils from the rectangula lever group displayed instinctive drift here also.

The lower portion of Figure 1 presents a breakdowı of the PRP data based upon the predominant responsi topography displayed by each animal. The four gerbil that maintained the shaped response showed systemati increases and decreases in PRP duration as a function o changes in the FR requirement. These results are con gruent with the findings for other species (Ferster $d$ Skinner, 1957; Powell, 1972). On the other hand, the 1( animals displaying instinctive drift showed little chang in pause duration as a function of changes in the re sponse requirement. Vanderweele, Abelson, and Tellisl (1973) reported a similar finding with gerbils, but the did not present a quantitative analysis of their results

Figure 2 presents an analysis of the response rate cal culated independently of the PRP (number of response divided by session time minus PRP time). This is some times referred to as the running rate. The upper portion of the figure shows that the response rate generally in creased for the animals in each group as the FF increased to 40 . However, the rate continued to increas as the FR decreased to 25 and 15 , but then decliner with further reductions in the ratio requirement. Thi apparent "overshooting" may have occurred because o the relatively brief time each ratio was in effect (fiv sessions), so that sensitivity to schedule changes wa delayed. The lower portion of the figure again presents breakdown of the animals into two groups based of their predominant response topography. The 10 animal that displayed instinctive drift maintained a substantiall: 


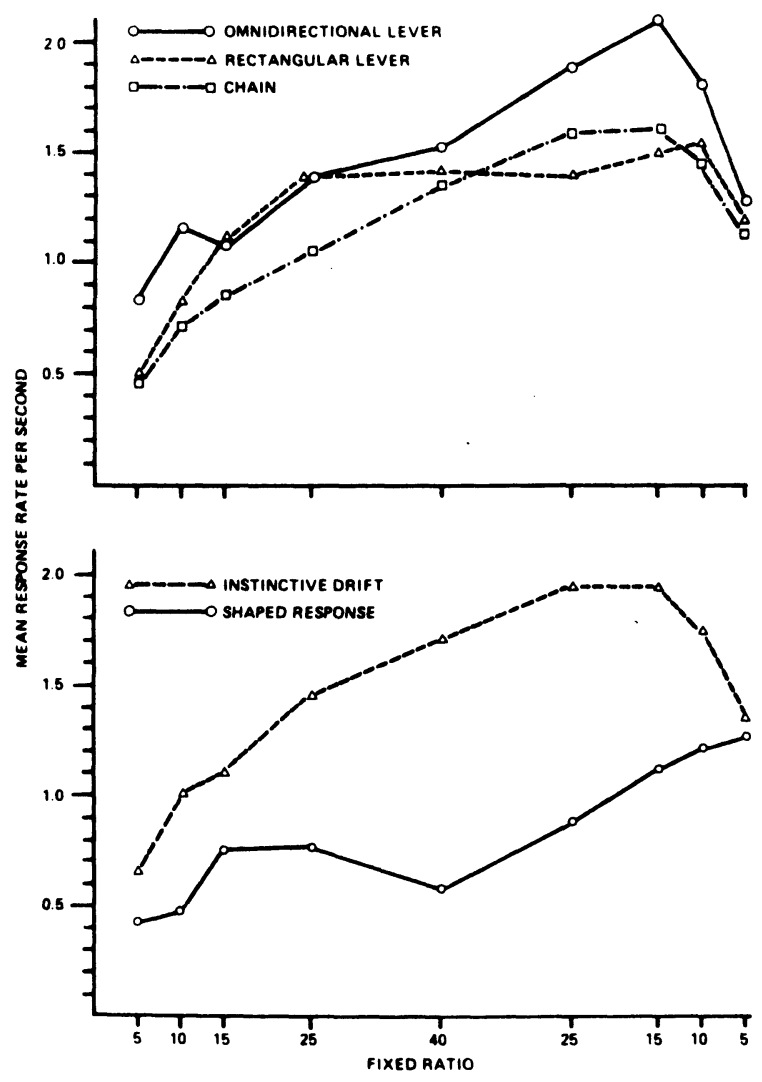

Figure 2. Upper portion: The mean response rate, calculated independently of the postreinforcement pause, for each manipulandum group as a function of the FR requirement. Each data point represents the mean performance over the final three sessions at each FR. Lower portion: The same data as presented above except that the gerbils are divided into two groups based upon whether the animal showed instinctive drift or maintained the leverpress response.

higher response rate, except for the final ratio, compared to the four gerbils that maintained the shaped response. In addition, the shape of the curves for the two groups appeared slightly different. The curve for the "instinctive drift" animals is quite similar to that just described, whereas the animals that maintained the operant response showed a gradual increase in response rate over the entire course of the experiment.

\section{EXPERIMENT II}

This experiment was intended to assess the generality of our earlier results using a completely nondomesticated species, the cotton rat. It was reasoned that the displacement of operant behavior by more genetically based responses should be even more prominent in a species which had not been exposed to domestication. Mongolian gerbils have been bred in captivity for approximately 20 years (Monroe, 1967), so it may be claimed that the species is partially domesticated. Cotton rats (Sigmodon hispidus) and gerbils (Meriones unguiculatus) are rather closely related, both belonging to the same order (Rodentia), suborder (Myomorpha), and family (Cricetidae).

\section{Method}

Subjects. Five cotton rats, two males and three females, were used. The rats were the first generation offspring of field-trapped adults. They were between 6 and 11 months of age at the start of the experiment and their weights ranged from 80 to $112 \mathrm{~g}$. All rats were experimentally naive and were maintained at approximately $80 \%$ of their normal weights during the experiment.

Apparatus. The apparatus was the same as in Experiment I, except that only the rectangular lever was employed.

Procedure. The cotton rats were first shaped to press the lever with two paws, and all rats successfully acquired the response. Then the rats were exposed to the following series of increasing FR requirements: 5, 10, 15, 25, 40, 60, 90. Two of the rats were then studied as the FR decreased to 60 and then 30 , while the remaining three rats were studied at FR 40 only during the decreasing series. Each ratio requirement remained in effect for a particular rat until responding stabilized. The stability criterion required that response rate remain within a range of $\pm 10 \%$ of the mean rate over three consecutive sessions. The duration of a session was based on the time it took each cotton rat to obtain 34 reinforcers.

\section{Results}

There was clear evidence of instinctive drift for each cotton rat by the time the FR increased to 15 . That is, the shaped leverpress was permanently replaced by a response topography in which scratching and biting of the lever was prominent. In addition, four of the rats frequently grasped the lever in their teeth and vigorously shook their heads in the vertical plane.

The mean postreinforcement pause for the group was relatively constant from FR-5 to FR-60, but then increased substantially at FR-90, as shown in Figure 3. When the FR requirement decreased, the mean pause durations decreased also, to values which approximated those obtained during the increasing series. The reversibility shown here demonstrates that ratio size did influence pause duration, but only when the value of the former was rather large.

Figure 3 shows also that the mean response rate for the cotton rats, which was calculated as described earlier, increased gradually as the ratio requirement increased. The curve for the group appears to be a negatively accelerated increasing function. The response rates obtained with decreasing ratio requirements closely approximated those obtained during the increasing series, so there is further evidence of functional control.

\section{GENERAL DISCUSSION}

The most significant finding here was the occurrence of instinctive drift in almost all animals studied, with exposure to FR schedules of food reinforcement. Our results appear similar to the observations of Breland and Breland (1961), in that an arbitrary operant response was displaced by responses which appear to be genetically based and are more prepotent in the animal's behavioral repertoire. While the appearance of instinctive drift was associated with increases in the number of responses required to obtain food, in both our study and the 

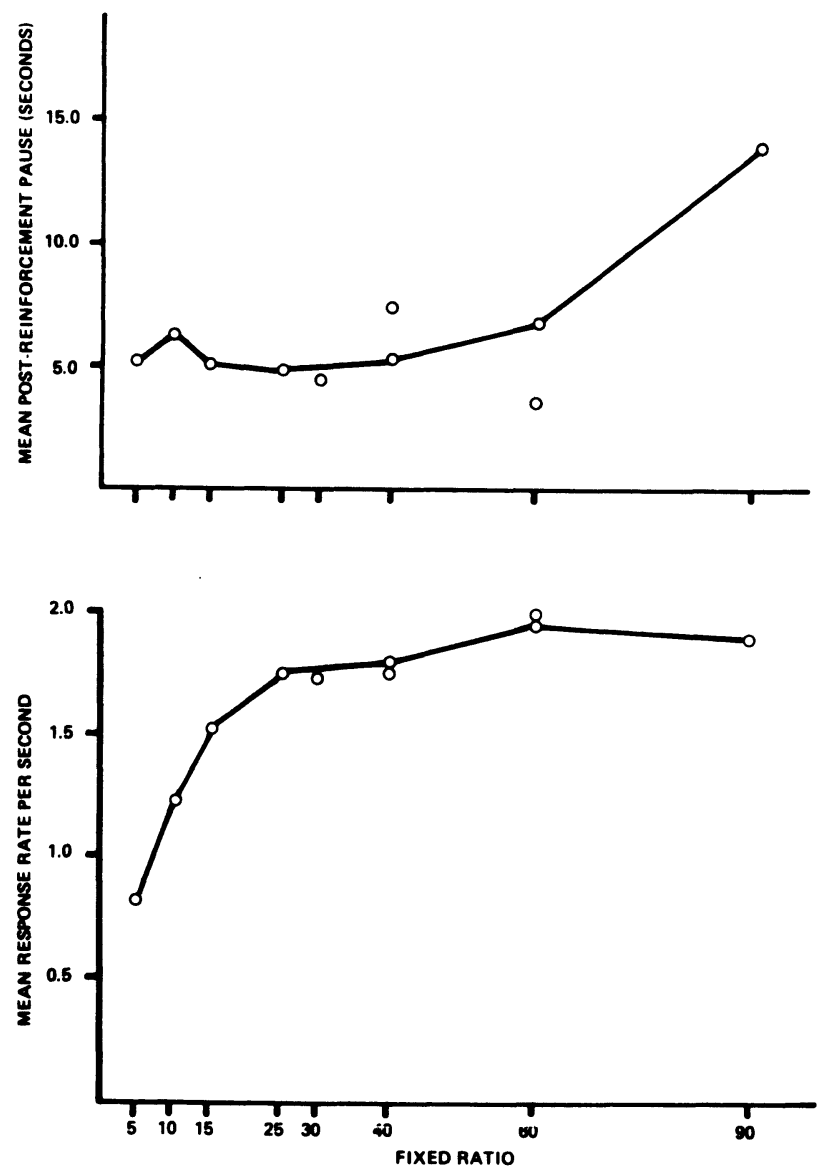

Figure 3. The mean postreinforcement pause and response rate for the cotton rats as a function of the FR requirement. The unconnected data points were obtained during the decreasing series.

Breland's, this may not have been a causal factor. This possibility clearly exists, based on our observation that once the leverpress was displaced during the increasing series, it never reappeared during subsequent sessions, including those in which the ratio requirement decreased.

While the Breland's reported that the occurrence of instinctive drift resulted in less frequent reinforcement, our findings were essentially the opposite. Either outcome seems to be possible, when considered logically, for the rate at which the subject obtains reinforcement will be determined by the interaction between the topography of the genetically based behavior which develops and the form of the operant response specified by the experimenter. So the occurrence of instinctive drift need not involve a conflict between explanations of behavior based on genetic mechanisms, as opposed to explanations which stress reinforcement as derived from the Law of Effect. It is possible, as seemed to occur here, that genetically based behaviors may be elicited which facilitate the acquisition of reinforcers.

The finding that animals which developed instinctive drift did not display the typical functional relationship between FR size and postreinforcement pause duration, appears to be noteworthy. This suggests that with the occurrence of instinctive drift, the behavior of the animal becomes less sensitive to the prevailing contingencies of reinforcement-a point stressed heavily by the Breland's. However, some sensitivity does remain, as shown by the marked increase in the pausing of the cotton rats at FR-90, and the subsequent decrease which occurred when the FR decreased. This result seems indicative of a process involving dual control by genetic and environmental factors, in which control may be shared or one factor may override the other, depending upon their relative potency.

Analysis of response rates shows that, in general, rate increased with increase in FR size, at least up to FR-40. The continued increase in rate when FR decreased, which was found in Experiment I, probably is attributable to the small number of sessions each FR remained in effect. When responding was studied until it stabilized at each FR, as in Experiment II, this effect did not occur. While the direct relationship found here between response rate and FR size is not unique (Barofsky \& Hurwitz, 1968), an inverse relationship has been reported more frequently (Felton \& Lyon, 1966; Powell, 1968, 1970, 1972).

The present research shows that a phenomenon identified as instinctive drift may intrude into experiments involving appetitive reinforcement. This may be more likely to occur when one is studying nondomesticated animals. When instinctive drift does occur, it seems to alter substantially the functional relationships that have typically been observed under similar conditions. While our research does not identify the causes and boundary conditions of this phenomenon, these appear as worthy goals to pursue in future investigations.

\section{REFERENCES}

Barofsky, I., \& HuRwitz, D. Within-ratio responding during fixed-ratio performance. Psychonomic Science, 1968, 11. 263-264.

Bolles, R. C. Reinforcement, expectancy, and learning. Psychological Review, 1972, 79, 394-409.

Breland, K., \& Breland, M. The misbehavior of organisms. American Psychologist, 1961, 16, 681-684.

Felton, M., \& Lyon, D. O. The post-reinforcement pause. Journal of the Experimental Analysis of Behavior, 1966, 9. 131-134.

Ferster, C. B., \& SKInNer, B. F. Schedules of reinforcement. New York: Appleton-Century-Crofts, 1957.

Monroe, B. N. Gerbils. Jersey City, N.J: T. F. H. Publications, 1967.

PowELL, R. W. The effect of small sequential changes in fixed-ratio size upon the post-reinforcement pause. Journal of the Experimental Analysis of Behavior, 1968, 11, 589-593.

Powell, R. W. Probability of reinforcement and fixedratio responding: A model. Psychological Record, 1970, 20, 73-80.

Powell, R. W. Responding under basic schedules of reinforcement in the crow. Journal of Comparative and Physiological Psychology, 1972, 79, 156-164.

Staddon, J. E. R., \& Simmelhag, V. L. The "superstition" experiment: A reexamination of its implications for the principles of adaptive behavior. Psychological Review, 1971, 78, 3-43.

Vanderweele, D. A., Abelson, R. M., \& Tellish, J. A. A comparison of ratio behavior in the gerbil and white rat. Bulletin of the Psychonomic Society, 1973, 1, 62-64.

(Received for publication May 13, 1976.) 